\title{
FROM LIDAR DATA TOWARDS HBIM FOR STRUCTURAL EVALUATION
}

\author{
R. Argiolas ${ }^{1}$, A. Cazzani ${ }^{1}$, E. Reccia ${ }^{1}$, V. Bagnolo ${ }^{1, *}$ \\ ${ }^{1}$ DICAAR, University of Cagliari, Faculty of Engineering and Architecture, Cagliari (CA), Italy - raffaele.argiolas@ hotmail.com \\ (antonio.cazzani, emanuele.reccia, vbagnolo)@unica.it
}

\author{
Commission II, WG II/8
}

KEY WORDS: HBIM, Point Clouds, 3D Modelling, Algorithmic Modelling, Stellar Vaults, Finite Element Model

\begin{abstract}
:
In HBIM processes, the extraction of geometric components from 3D point clouds data can sometimes be a complex process. The socalled "Scan to BIM" process has been widely utilized: deriving 3D models from point clouds often a local modelling of geometric components is necessary. This leads in most cases to use external modelling tools or complex local modelling processes. In both cases, we often get a model that cannot be reused for other items belonging to the same category, contravening the BIM philosophy. Vaulted systems are a typical example of complex elements that we can find in historical architecture. The paper presents the first results of an ongoing research on geometric modelling and structural evaluation of masonry ribbed vaults. An algorithm is developed to generate a NURBS surface of masonry vaults that, starting from the data extrapolated from the point cloud, allows to obtain an HBIM family. The research aims to overcome the inability to reference to standardised objects in local modelling of historical architecture elements. Directed to a standardization in the geometric modelling process of 3D laser scan data, the developed workflow is a possible alternative to commonly used workflows. Particular attention is focused on a case study of stellar vaults, a special class of masonry ribbed vaults whose three-dimensional geometry features a star-shaped projection on the horizontal plane. The work is carried out to verify that this family can be used for the structural analysis of stellar masonry vaults.
\end{abstract}

\section{INTRODUCTION}

\subsection{Towards a HBIM family of stellar vaults}

Using laser scanning-based surveying techniques to collect 3D morphological data of historic architectures, geometric modelling commonly involves the transition from point clouds to surface models. In HBIM workflows, it is not always possible to use object libraries and families with parametric constraints and properties, especially in the presence of complex elements. The extraction of geometric information from LiDAR data is not always a simple process and sometimes, not being able to rely upon standardized objects, the geometric components are derived locally. In these cases, the so-called Scan-to-BIM process is commonly used.

The inability to reference throughout standardised objects can hinder a meaningfully or efficiently application of BIM workflows. To overcome this drawback, a new workflow has been designed. Aimed at automate the geometric modelling process of 3D laser scan data, the developed workflow is a valid alternative in cases where local modelling is required. The procedural modelling offers us an alternative way to build local models starting from segmented point clouds.

Algorithms allows us to parameterize the geometric modelling process to obtain NURBS models of the detected surfaces (Fig. 7). If necessary, the process involves a further step which allows the conversion of NURBS surfaces in 3D mesh models (Fig.8). The advantage of the proposed workflow lies in overcoming the underperforming assets of local models in supporting the evaluation of performance of historic buildings. Specifically, the workflow lends itself better to application in category of objects not natively present in the pre-compiled libraries of parametric objects.
An interesting example of such categories of objects is that of vaulted systems. Frequently recurring in historical architecture, vaulted systems are elements that commonly require careful surveying and modelling processes, which often refers to comparisons and checks with the models proposed by historical treatises.

\subsection{Integrating structural evaluations}

In the study of vaulted systems, it is often necessary to proceed with structural evaluations. The development of an algorithm aimed at the generation of NURBS surfaces of masonry vaults gives us the opportunity to test the obtained model for the structural analysis of stellar masonry vaults.

The research develops various structural analyses based on the NURBS model obtained with the algorithm. The results of the structural analyses were then compared with those obtained through a commonly used methodological approach to surface modelling.

\section{GOTHIC STELLAR VAULTS}

\subsection{Case study}

The stellar vaults are a complex vaulted system typically adopted in Gothic architecture. As elsewhere in the Mediterranean area, also in Sardinia this kind of vaults are often found in religious constructions built on the island between the 14th and 17th centuries. In church architecture, the stellar vault can resolve the covering of different parts of the building, such as the side chapels. In the ecclesiastical architecture of Sardinia, stellar vaults are often adopted as a solution for the presbyterial area at the so-called "capilla mayor" (Casu, 2013). 
Even the stellar vault of the considered case study occupies the position corresponding to the original presbytery of the church. It consists of a ribbed vault system with five keystones, set on a quadrangular plan, which concluded a sequence of ribbed cross vaults starting from the entrance portal and covering the nave. The position of the five keystones corresponds to that obtained with the geometric trace in Fig.1.

\subsection{Geometric assessment}

The standardization of models collected in architectural treatises, historically consolidated in the design practice and in construction on site, introduces graphical schemes and parameters necessary for the design and erection of vaulted systems.

The hierarchical structure implementing the transition from simple to complex vaulted systems, defines an additional theoretical reference and a standard on which we can rest the algorithmic modelling. The stellar vault is a particular type of Gothic ribbed vault. It can be considered an evolution of the simple cross vault, presenting a more complex geometry and a characteristic system of auxiliary ribs.

According to the rules of the three arches explained by Villard de Honnecourt in the Livre de Portraiture, the backbone of the vault can be designed using the same circle (Capone, 2014). In his construction, Villard obtains round diagonal arches and pointed side arches. Complying with this practice, the system of the stellar vault of Santa Maria del Monte is clearly set on the geometry of the circle but, unlike the rule proposed by Villard de Honnecourt, the two diagonal ribs consist of two centered arches (pointed arch) (Fig.2). Furthermore, by presenting different curvature values, in our case study the curvatures of the different arcs cannot be traced back to a single radius value as in the three-arch rule defined by Villard.

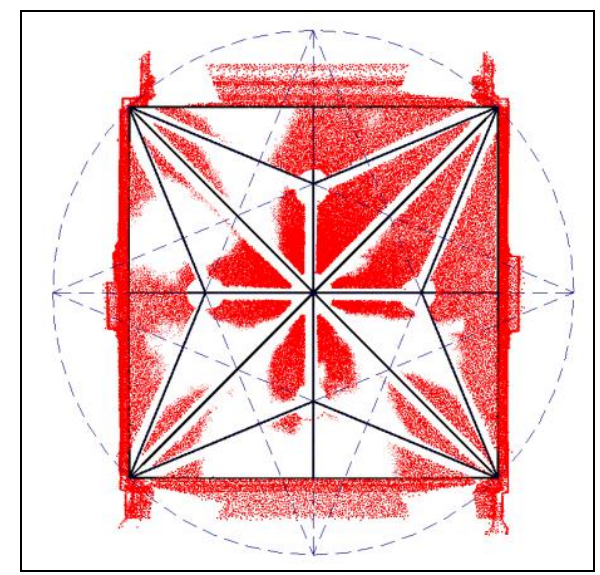

Figure 1. Geometric trace of stellar vault in plan

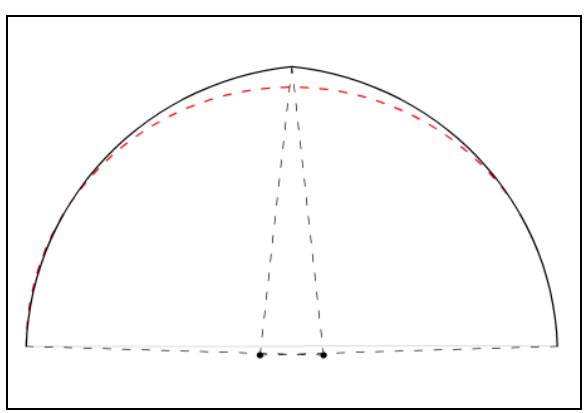

Figure 2. Comparison between the pointed diagonal arch of the stellar vault in Santa Maria del Monte (black) and the theoretical round diagonal arch.

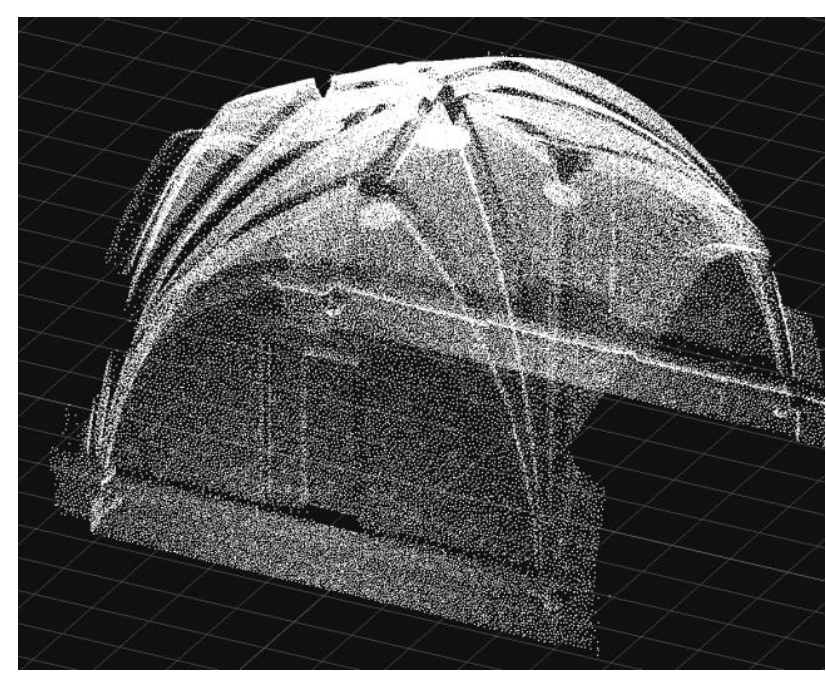

Figure 3. 3D point cloud of the stellar vault

\section{3D MODELLING IN BIM ENVIRONMENT}

\subsection{HBIM workflows}

In the normal implementation of the BIM methodology for historical heritage, the modelling of particularly complex elements generally takes place in a local way, that is to say, creating a single object specific to the case examined. Starting from the point cloud (Fig. 3) it is possible to obtain a direct mesh surface which, at the expense of speed and file size, offers relatively high accuracy; the mesh thus obtained can then be imported as an object within the BIM environment and become part of a parametric family, but as a non-modifiable object (Barazzetti et al., 2015).

However, the point cloud can also be appropriately sectioned to obtain profiles that can be used as a basis for modelling NURBS surfaces (Biagini et al., 2016), often generated with modellers outside the BIM environment and then imported into it; also in this case we speak of fixed elements that are inserted into parametric families whose variations will however concern the other elements present. The choice to use external tools is dictated by the fact that in most cases the modelling tools offered natively by BIM software do not offer the flexibility and potential necessary for the development of complex surfaces (Oreni et al., 2014; Dore et al., 2015).

In the rare cases in which it is possible to obtain all 3D models in the BIM environment, these are often non-parametric, or only in part, making a profound intervention on the geometries necessary if the model is to be modified, or again they are useful in a very strict contest (Baik et al., 2014).

In all the three processes mentioned a factor remains constant: with each new element to be modelled, although with geometries and shapes similar to the initial object, it will be necessary to repeat the whole modelling process from the beginning (Fig. 4).

In the proposed study, an alternative workflow was applied which entrusts all the steps of object creation to the algorithmic modelling using the Dynamo software within Revit. This allowed us to define an algorithm able to model the object studied with the advantage, however, in the case of similar objects, of being able to reuse the same algorithm with the only burden of entering the new initial parameters. So if historical objects in general are out of the BIM logic of the reusable parametric family, in our workflow it is their genesis to be both 
parametric and reusable, bringing these elements back into full BIM philosophy (Fig. 5).

\subsection{The theoretical model of Villard de Honnencurt}

The most accredited construction for stellar vaults is that proposed by Villard de Honnecourt (XIII century) which provides, starting from the planimetric scheme, the determination of the curvature that all the arches will share. This curvature is that of the diagonal arc having a semicircular profile; it is therefore as understandable as a building with a regular square base, like for the vault in Santa Maria del Monte. It is then a matter of following when indicated by Villard, obviously taking the liberty of simplifying those passages that have shortcuts given by the use of the assisted drawing. We then go on to determine the base square and its diagonal and on this we create the round arch that represents the ogive of the vault; since the construction foresees that the tiercerons are in an intermediate position between ogives and lateral arches, the ogive obtained is copied by rotations of $22.5^{\circ}$ and $45^{\circ}$. The three arcs thus obtained are then cut according to a $\mathrm{YZ}$ plane passed through the origin and their tops united for the creation of the liemes. All that remains is to close the surfaces delimited by arches and liemes to obtain a segment of the vault according to the ideal model; since finally the ideal model provides a perfectly regular and symmetrical vault, a series of mirrors and rotating copies is sufficient to complete the vault.

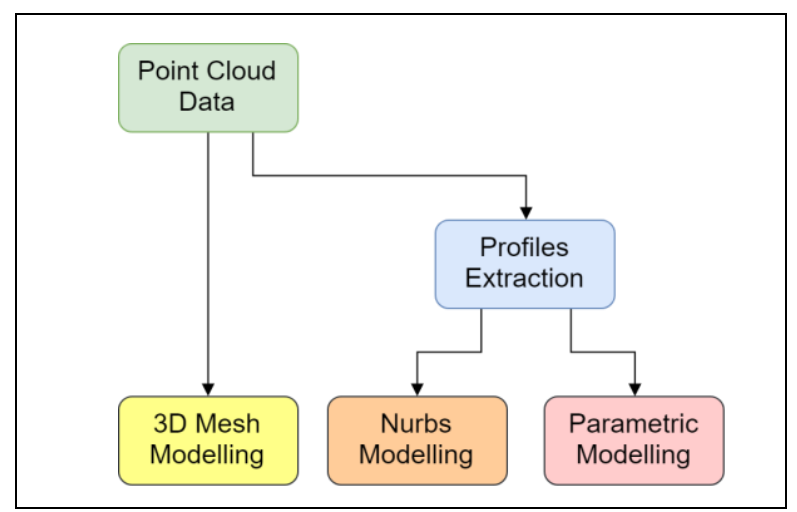

Figure 4. In common workflow every new object requires a new modelling process from the scratch

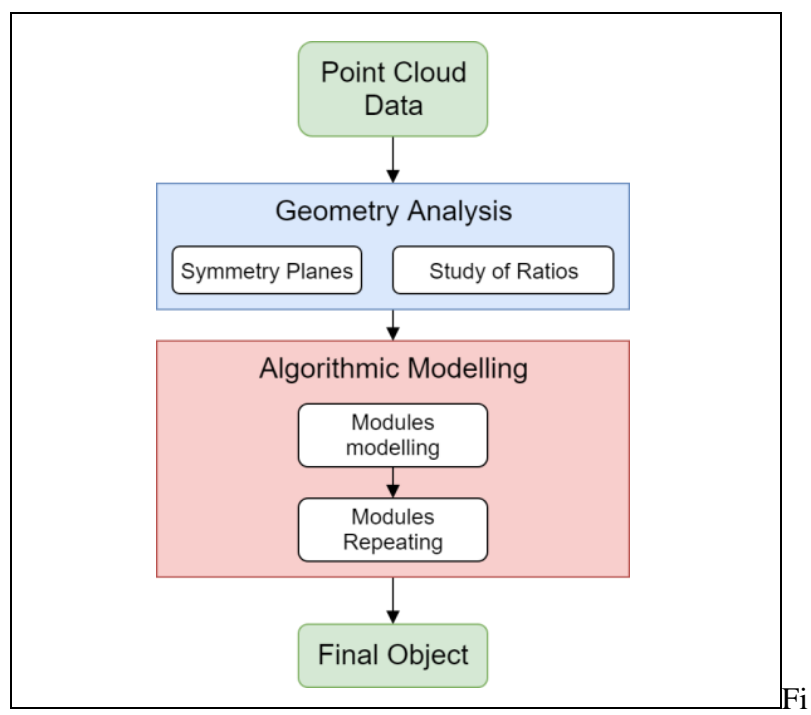

Figure 5. A single algorithm can generate several models just changing input parameters

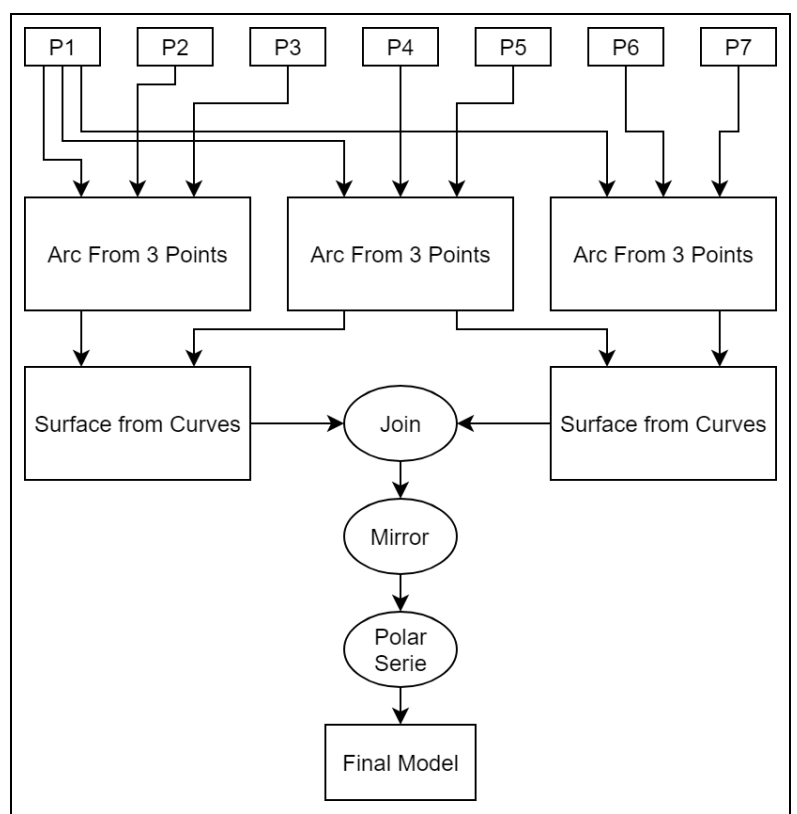

Figure 6. Diagram of the algorithmic modelling process of the stellar vault

\subsection{From the theoretical model to the algorithm coding}

However, this "ideal" model is inconsistent with the real model, a sign that the ideal model was not followed for the construction of the Santa Maria del Monte vault, but it was decided to build once with a raised cone, thus not having a corresponding profile to a monocentric round arch but rather an arc with two centers. This inevitably implies that the algorithm based on the construction of Villard must be reworked and made more flexible with respect to the rigid sequence of steps derived from the treaties.

A first change that was decided to apply was to replace the arches with constant curvature with three arches with variable curvature, drawn according to the two-center arches scheme; the input data in this case will be 3 points for which to pass each bow, the first at the shutter, the second intermediate and the third in the key; instead, the symmetry restraints of the vault and its square setting are currently maintained.

This means that for the complete modelling of the vault a very limited number of points is needed from which the whole generation of the model derives; measurement operations are performed directly on the point cloud (Fig.6). The model thus obtained appears much more corresponding to the point cloud obtained with the laser scanner; we can then move on to a more accurate comparison to understand how really the two models are similar.

A first check is that of the sections: we proceeded with the sectioning of the point cloud in a series of sections with a constant pitch (about $10 \mathrm{~cm}$ ), trying to keep the depth of the sections as small as possible so as to prevent the presence of too many overlapping points could generate data disturbance; we then performed an interpolation of the points and their approximation by means of polylines. Finally, a cloud of points and sections were imported into Revit and aligned to the model of the vault for comparison. It was therefore sufficient to set up a series of sections of particular interest, at a reduced depth, and to make a comparison between the various models.

In this phase, one of the great advantages of working in an integrated manner in the BIM environment was evident: if in fact commonly the model is created externally and then imported, with the obligation therefore to repeat the procedure 
for each modification, Dynamo allows to model dynamically, always staying in the BIM environment and also offering a sort of "preview" of what we are creating. This therefore allows us to act on the initial parameters in real time if we become aware of an incongruity, or even to modify part of the algorithm and immediately see the results to evaluate the validity of the interventions. When a difference in height between the model and the cloud was found, given by an incorrect measurement on the point cloud, the model could be corrected in real time to ensure that it was as close as possible to the point cloud.

\subsection{Comparison of the algorithmic model with 3D point cloud}

The NURBS model, derived from the algorithmic modelling, compared with the point cloud profiles has led to satisfactory results. The CloudCompare software allows us to quantify the average deviation and the classification of the points. The results are shown as chromatically characterization of correspondences and discrepancies. In addition, the displayed offset is oriented, so it is possible to know negative and positive shifts (Fig. 9). From the histogram generated by CloudCompare, we can see how the great majority of the points depart from the model for values included between $-3.5 \mathrm{~cm}$ and $3.5 \mathrm{~cm}$ (Fig.10).

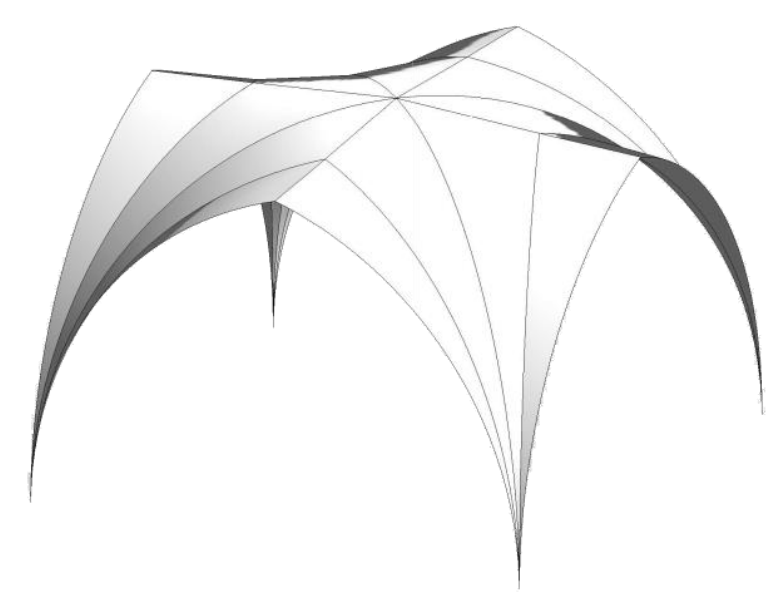

Figure 7. NURBS model deriving from the algorithmic modelling.

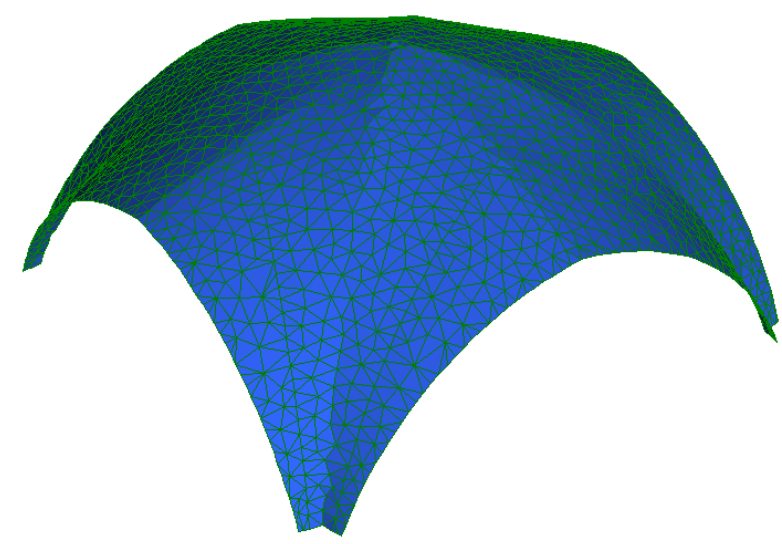

Figure 8. 3D mesh model deriving from the algorithmic modelling.

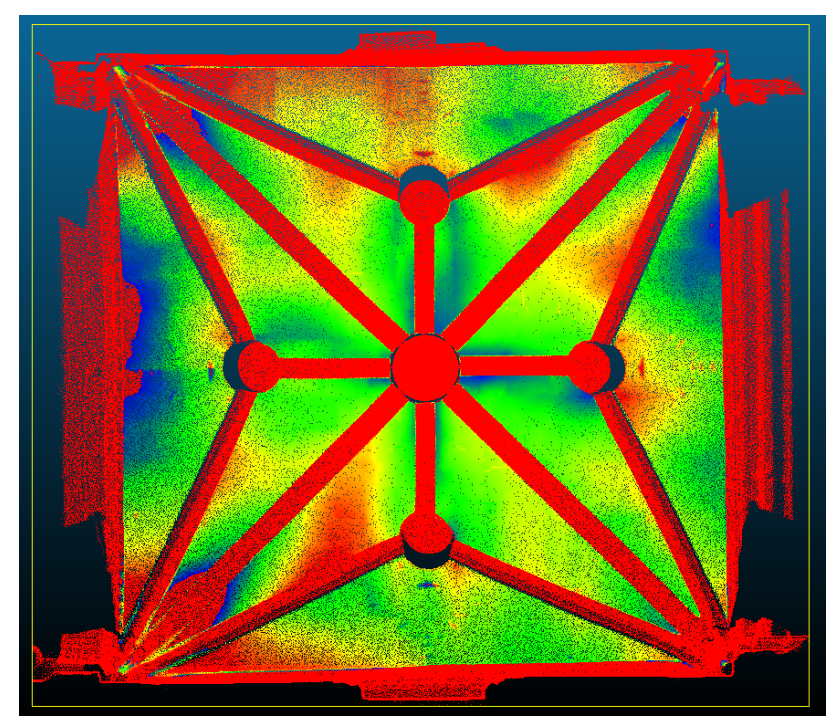

Figure 9. Chromatic scale showing the distances between the point cloud and the surface from the algorithm. The reddish color values show a positive shift (up) compared to the NURBS point cloud, while the chromatic values in blue show the negative deviations (downwards).

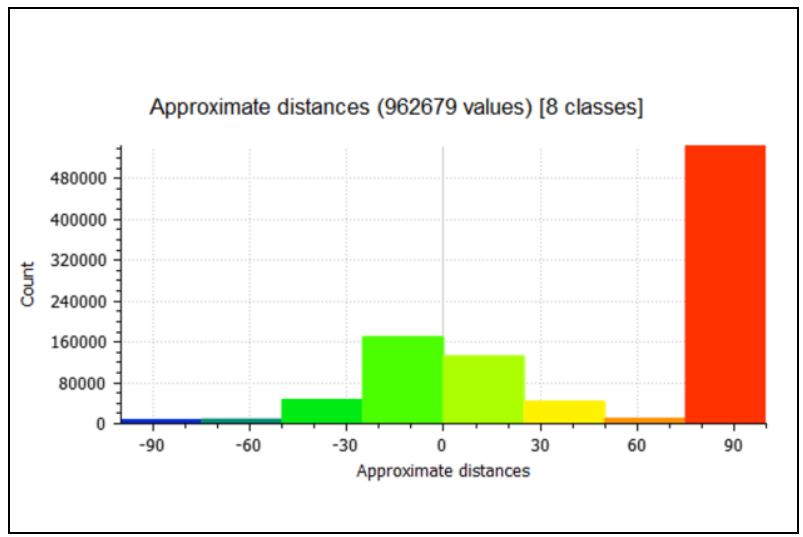

Figure 10. Histogram generated by CloudCompare software showing the classes of the values of the distances between the point cloud model and the NURBS surface defined by algorithmic modelling.

In the overlapping ribbing points and keystones are not taken into account as they are not evaluated in the structural assessment model; so, these points, are highlighted with a very bright red color, indicating the strong distance from the model. The comparison presented here certainly gives us an idea of how close our model is to reality, but we need to understand whether the measured differences may or may not be acceptable for our purposes, which we recall are the static verification of the vault and its comparison with what obtained by local modelling. The next step then is to compare our deviations with those relating to the point cloud with respect to the NURBS model obtained on Rhino from the previous DICAAR works (Manconi, 2016). To do this it is sufficient to re-execute the same operations, making up to our model the one modelled locally; this modelling was carried out by tracking the dimension lines of the point cloud on which the NURBS surfaces were then created (Fig. 11). In this second case the relative histogram was also generated (Fig. 12). 


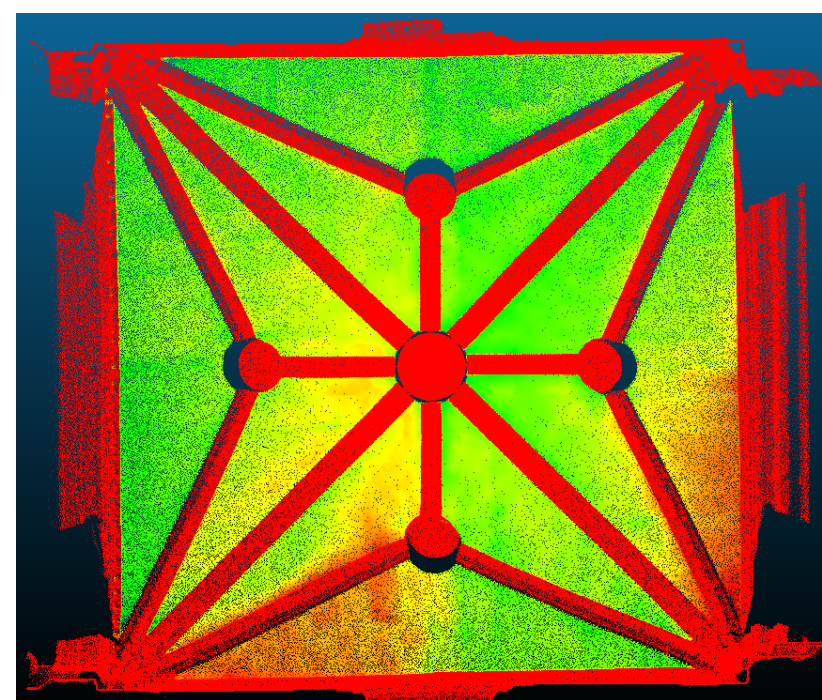

Figure 11. Chromatic scale showing the distances between the point cloud and the NURBS.

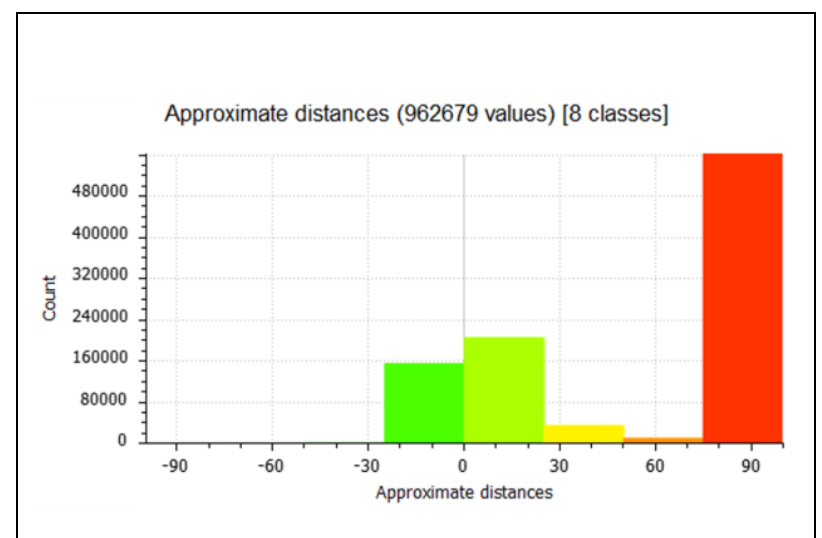

Figure 12. Histogram generated by CloudCompare software showing the classes of the deviation values between the point cloud model and the NURBS model.

It is immediately evident from the images how the local model has a greater degree of precision and how the discrepancy is more limited compared to what happened with our model, a result that in any case we expected as we know well the local modelling made ad hoc for a specific case in the face of greater expenditure of time, it offers greater relevance to reality. One of the aspects that must in fact be evaluated is certainly the gap between the two solutions, but also taking into account the large differences in time and work that the two methods provide.

However, since one of the purposes for which the model is generated is that of static verifications, it will be on the results of these that the validation of the algorithmic model will take place, thus decreeing its validity as an alternative to local modelling.

\subsection{Mesh treatment for structural evaluation}

The starting point for mesh generation is the NURBS surfaces obtained, in our case, by algorithmic modelling and, in the case of comparison, by modelling on Rhino. The latter are modelled through the analysis of the intrados, therefore they have the support points shaped according to the course of the ribs; in our case, on the other hand, starting from the "ideal" model, the supports are represented by surfaces that converge in a point, as happens in the average surface. However, this type of support has the disadvantage of needing very deformed and dense triangles to be represented; this generally creates problems in the analysis phase, moreover these convergences are not very useful for the analysis purposes, it was therefore decided to cut the final ends of the model. Once this is done, the next step was creating the 3D mesh surface; to do this, Dynamo provides a library of appropriate tools for managing meshes. In particular, we have used the creation of meshes from geometries; this block allows, once defined the tolerance and a limit of edges, to construct a mesh surface that follows the starting surface. The mesh thus created is not yet the definitive one, in fact it could have areas of different polygonal density, and possible opening points; to overcome this problem a re-meshing operation can be carried out, that is a redistribution of the triangles so that the coverage is more homogeneous.

This procedure also allows us to homogenize the proportions of the triangles, avoiding future problems given by dimensional relationships of the too disproportionate triangles, a nonnegligible factor during finite element analysis. The meshes thus obtained are exported in the STL format, generally well managed by the FE software; it is important to aim at having mesh models with the most similar characteristics possible, especially in terms of triangle number and homogeneity in their distribution, since in this way it is possible to exclude any discrepancies given by a configuration too different between the models to be analysed and compared.

Once we get the meshes according to the features described so far we can start setting up the files structure evaluation.

\section{STRUCTURAL EVALUATION OF GOTHIC STELLAR VAULTS}

\subsection{Finite Element Model}

Two Finite Element Models (FEM) have been realized in order to compare the two meshes by means of structural evaluation. At this phase, the elastic behaviour is investigated. The vault is represented through its reference surface, fit to perform threedimensional membrane analysis. The thickness of the vault could not be measured directly, therefore a thickness equal to $15 \mathrm{~cm}$ has been assumed, that is the minimum thickness provided by historical treatments (Viollet-le-Duc, 1854-1868) and scientific literature for this typology of masonry vault Como, 2010).

The vault has been supposed constrained only at the base according to the hypothesis of very low connection with lateral walls, which are located only on two sides.

The vault is entirely built with cut stone, the structure appears generally regular, with brick rows orthogonally aligned with the perimeter arches and mortar lime joints with an average thickness equal to $2 \mathrm{~cm}$ in the webs.

For mechanical properties of masonry material, we refer to the work (Grillanda et al., 2019), in which accurate numerical analyses of the Santa Maria del Monte stellar vault have been performed. Masonry is modelled as an isotropic homogenized continuum, the mechanical properties adopted are:

- Young's modulus: $\mathrm{E}=2800 \mathrm{MPa}$;

- Poisson's coefficient: $v=0.2$;

- specific weight: $\gamma=22 \mathrm{kN} / \mathrm{m}^{3}$;

which are in good agreement with values suggested by technical literature (Cuccuru et al., 2012).

Being here the ribs and keystones not considered in the mesh, the analysis is performed with reference to the previous work (Manconi, 2016). 
The models have been realized importing the two meshes in a commercial FE code, namely STRAUS7, and are made by triangular shell elements. Number of nodes and elements of the two models are reported: algorithm model consists of 1632 nodes and 2982 plates, while NURBS model of 1541 nodes and 2844 plates.

\subsection{Structural assessment}

Linear static analysis under self-weight load has been performed to evaluate the differences in the structural behaviour between the two models. A comparison of some of the results obtained is reported, in particular attention is focused on vertical displacements and principal stresses.

Figure 13 shows the vertical displacements (DZ) for the two models subjected to self-weight load. It is possible to notice that the results are good agreement, differences of a few tenths of a millimetres.

In the graph reported in Figure 14, vertical displacements along the main diagonal are plotted for the two models: blue points refer to algorithm model while orange points to NURBS one. The two trends are very similar, with a slight rising at the haunch and lowering at crown. The differences are small - less than a millimetre - and mainly concentrated at crown.

The algorithm model is stiffer whit respect to the NURBS one: this is due to the fact that the geometry adopted in NURBS model is derived by the laser scanner survey of the vault intrados, while the algorithm model reproduces the middle surface of the vault

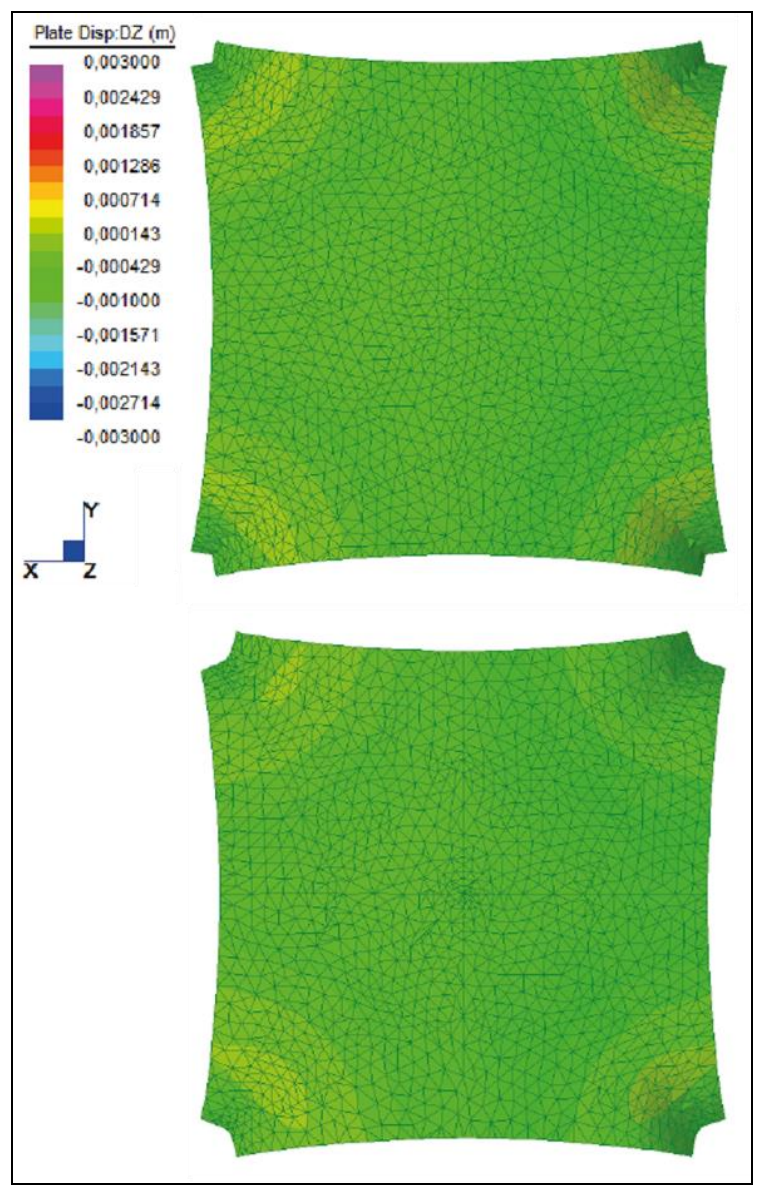

Figure 13. DZ displacement in the two different 3D mesh

models: on the left applied to the surface deriving from algorithmic modelling and on the right to the surface commonly derived from the point cloud

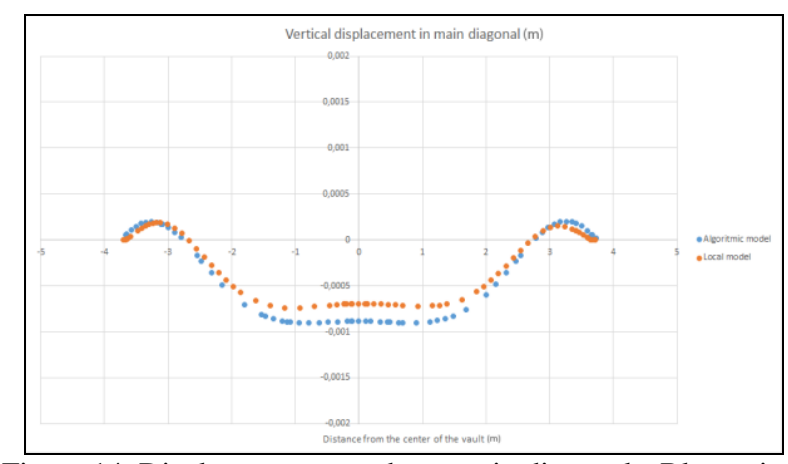

Figure 14. Displacements trends on main diagonals. Blue points refer to algorithmic model and orange ones refer to local model generated in Rhino.

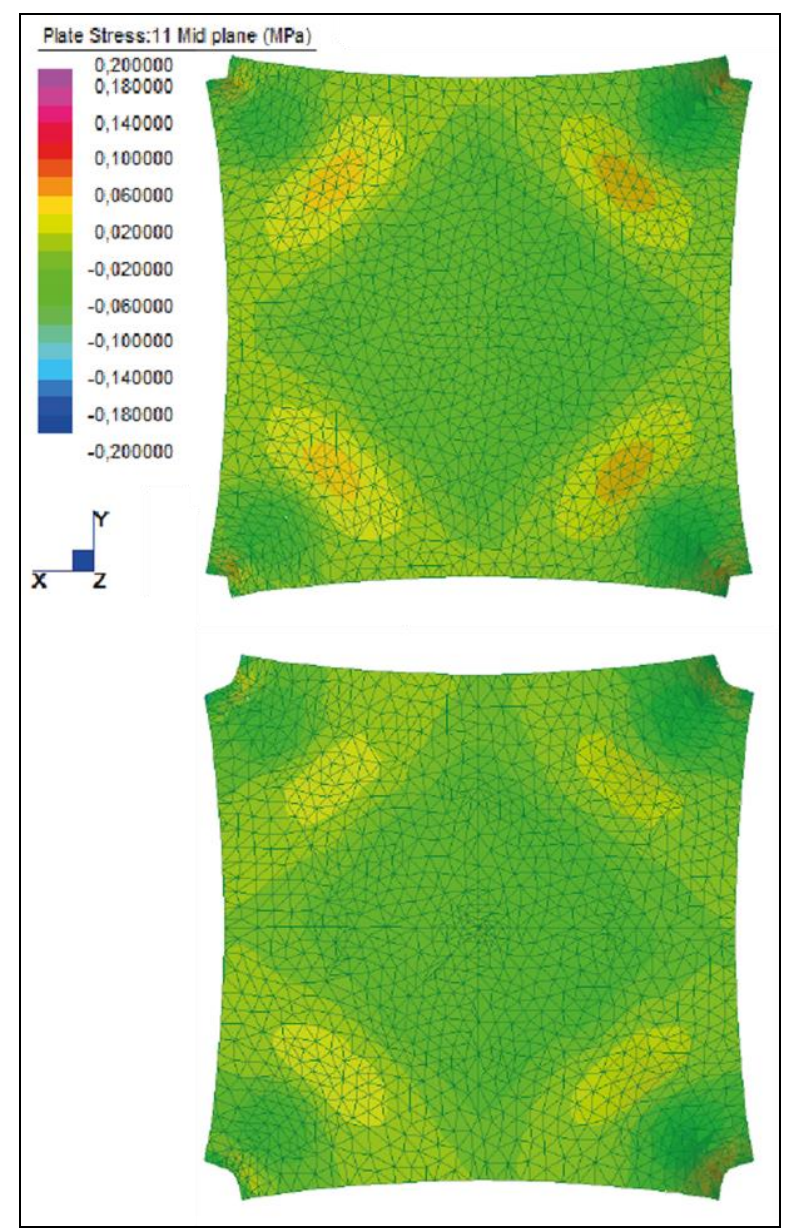

Figure 15. $1^{\text {st }}$ principal stress in the two different 3D mesh models: on the left applied to the surface deriving from algorithmic modelling and on the right to the surface commonly derived from the point cloud.

Pictures 15 and 16 provide contour plot of $1^{\text {st }}$ and $2^{\text {nd }}$ principal stresses for the two models. Also in this case, the results are good agreement, with small differences in the stresses distribution at haunches, related to the fact that the algorithmic model is perfectly symmetrical while the NURBS model has a slight asymmetry.

Comparison between the results obtained by the two models shows that the algorithmic model is able to describe the structural behaviour of stellar masonry vaults. More advanced structural analyses will be performed in order to validate the model and to highlight its limit of application. 


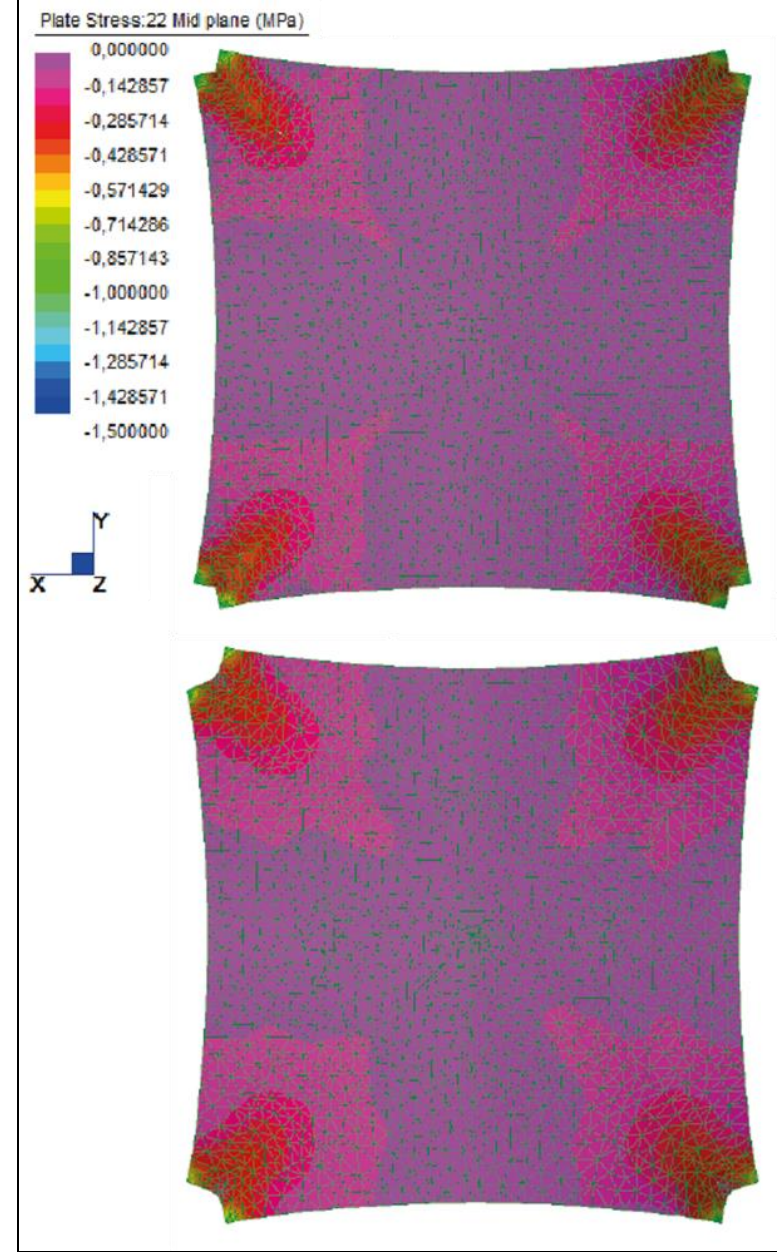

Figure 16. $2^{\text {nd }}$ principal stress in the two different 3D mesh models: on the left applied to the surface deriving from algorithmic modelling and on the right to the surface commonly derived from the point cloud.

\section{CONCLUSIONS}

The paper presents the first results of an ongoing research aimed at the study of alternative workflows for the local modelling of complex elements of historical architecture in a BIM environment.

The considered case study is the stellar presbytery vault of the church of Santa Maria del Monte in Cagliari. The analysis of this vaulted system represents the first step aimed at the study and calibration of a standardized process to be extended to other similar examples of vaulted systems. The purpose of research development will be to verify and refine the methodological approach tested in this first case study. Among the checks that we intend to conduct is the one related to the quality required for the point cloud to fulfill the results. In fact, for the purpose of a structural analysis, in our process the data derived from the "point cloud" useful in the computation of the algorithm are in a very limited number. With the analysis of other case studies, further confirmation may be obtained. The study will also be extended to other simple and complex vaulted system geometries.

\section{ACKNOWLEDGEMENTS}

The 3D laser scanning of the vault was carried out at LabMAST (Laboratory for historical and traditional materials and architectures), University of Cagliari, Department of CivilEnvironmental Engineering and Architecture (DICAAR). LIDAR point cloud product by Monica Deidda and Andrea Dessì.

Dr. Reccia fully acknowledges the research project funded by P.O.R. SARDEGNA F.S.E. 2014-2020 - Axis III Education and Training, Thematic Objective: 10, Specific Objective: 10.5, Action of the Partnership Agreement: 10.5.12, Call for Funding of Research Projects - Year 2017

\section{REFERENCES}

Baik, A., Alitany, A., Boehm, J., and Robson, S. 2014. Jeddah Historical Building Information Modelling "JHBIM"-Object Library. In: ISPRS Annals of the Photogrammetry, Remote Sensing and Spatial Information Sciences, Vol. II-5, pp.41-47.

Barazzetti, L., Banfi, F., Brumana, R., Gusmeroli, G., Previtali, M., and Schiantarelli, G., 2015. Cloud-to-BIM-to-FEM: Structural simulation with accurate historic BIM from laser scans. In: Simulation Modelling Practice and Theory, 57, pp.71-87.

Biagini, C., Capone, P., Donato, V., and Facchini, N., 2016. Towards the BIM implementation for historical building restoration sites. In: Automation in Construction, 71, pp.74-86.

Casu, P., 2013. Volte tardogotiche sarde: Disegno, misura, materia. UNICA, PhD thesis. A.A. 2011/12.

Capone, M., 2014. La discretizzazione della forma. Genesi e trasformazione: la geometria segreta dei reticoli spaziali delle volte gotiche. In: Disegnare idee immagini, anno XXV, 49, pp.36-48.

Como, M., 2010. Statica delle costruzioni storiche in muratura: Archi, volte, cupole, architetture monumentali, edifici sotto carichi verticali e sotto sisma. Aracne: Roma.

Cuccuru, F., Fais, S., and Ligas, P., 2014. Dynamic elastic characterization of carbonate rocks used as building materials in the historical city centre of Cagliari (Italy). In: Quarterly Journal of Engineering Geology and Hydrogeology, 47(3), pp.259-266.

Grillanda, N., Chiozzi, A., Bondi, F., Tralli, A., Manconi, F., Stochino, F., and Cazzani, A. 2019. Numerical insights on the structural assessment of historical masonry stellar vaults: the case of Santa Maria del Monte in Cagliari. In: Continuum Mechanics and Thermodynamics, doi.org/10.1007/s00161-019-00752-8.

Manconi, F., 2016. Analisi statica di volte a crociera nervate: La volta stellare della chiesa di Santa Maria del Monte in Cagliari. UNICA, unpublished master's degree thesis. A.A. 2015/16.

Dore, C.; Murphy, M.; McCarthy, S.; Brechin, F.; Casidy, C.; Dirix, E., 2015. Structural Simulations and Conservation Analysis -Historic Building Information Model. In: The International Archives of the Photogrammetry, Remote Sensing and Spatial Information Sciences, Vol. XL-5-W4, pp. 351-357.

Oreni, D., Brumana, R., Banfi, F., Bertola, L., Barazzetti, L., Cuca, B., and Roncoroni, F. 2014. Beyond crude 3D models: 
from point clouds to historical building information modelling via NURBS. In: Euro-Mediterranean Conference. Springer, Cham, pp. 166-175.

de Honnecourt, V., XIII sec. Villard de Honnecourt, Album de dessins et croquis. https://gallica.bnf.fr/ark:/12148/btv1b10509412z/f1.image (1 Jun. 2019).

Viollet-le-Duc, E.E., 1854-1868, Dictionnaire raisonné de l'architecture française du XIe au XVIe siècle. Édition Bance Morel: Paris. 